\title{
Necropotosis-Induciable Nanoliposomes for Enhanced Cancer Sonoimmunotherapy in Vitro
}

\author{
Meng-meng Li \\ Chongqing Medical University \\ Ya Zhu \\ Chongqing Medical University \\ Mi Yang \\ Chongqing Medical University \\ Yang Cao \\ Chongqing Medical University \\ Haitao Ran ( $\nabla$ ranhaitao@cqmu.edu.cn ) \\ Chongqing Medical University \\ Wei Zhang \\ Chongqing Medical University
}

\section{Research}

Keywords: necroptosis, reactive oxygen species, immunogenic cell death, sonodynamic, ovarian cancer

Posted Date: June 8th, 2021

DOl: https://doi.org/10.21203/rs.3.rs-560255/v1

License: (c) (1) This work is licensed under a Creative Commons Attribution 4.0 International License.

Read Full License 


\section{Abstract}

Background: Necroptosis has emerged as a therapeutic target for stimulating antitumor immune responses in dying tumor cells. However, its suppressed expression of receptor-interacting protein kinase 3 (RIPK3), a key enzyme in necrosis in most cancer cells, limits the clinical translation to exploiting necroptosis.

Design: We fabricated a multifunctional phase-transition nanoparticles platform by constructing Lip-ICGPFP-CRGD, utilizing liposome and indocyanine green (ICG) as the shell and perfluoropentane (PFP) as the core. The platform system represented the combination of sonodynamic therapy (SDT) and immunotherapy for cancer treatment by inducing necroptosis and disrupting the cell membrane through the acoustic cavitation effect mediated by ultrasound. In addition to their inherent contrasting ability under photoacoustic imaging, our liposomes may also be used as an ultrasound imaging probe after being irradiated with low-intensity focused ultrasound (LIFU).

Results: We demonstrate that nanoparticles can trigger necroptosis in ovarian cancer cells, which ruptures cell membrane by acoustic cavitation effect. When exposed to LIFU, the nanoparticles effectively facilitate the release of damage-associated molecular patterns by inducing burst-mediated cellmembrane decomposition. Moreover, the PFP phase change caused RIPK3/MLKL-independent necroptosis by acoustic cavitation effect, resulting in the release of biologically active DAMPs (CRT and HMGB1) to facilitate antitumor immunity. Therefore, necroptosis-inducible nanoparticles remarkably enhance antitumor immunity by activating $\mathrm{CD} 8^{+}$cytotoxic $\mathrm{T}$ cells and maturing dendritic cells in vitro.

Conclusion: We have successfully synthesized Lip-ICG-PFP-cRGD nanoparticles, which can achieve SDT and provoke necroptosis by bubble-mediated cell membrane rupture. The innovative nanoparticle causes immunogenic cell death in cancer cells via RIPK3-independent necroptosis, which is a promising enhancer for cancer immunotherapy.

\section{Introduction}

Over the last decade, ovarian cancer has become one of the most lethal gynecological malignancies with high incidence, poor prognosis, mortality rates, and resistance to conventional therapies. ${ }^{1-3}$ According to previous research, up to $30 \%$ of ovarian cancer patients die within five years due to relapse and distant metastatic development. ${ }^{1}$ Consequently, various approaches need to be explored to provide better clinical treatment. Chemotherapy and radiation are two conventional cancer treatments that can reduce cell viability and induce tumor cancer apoptosis by destroying cellular organelles and inhibiting cell division. ${ }^{4}$ Apoptosis has been considered as a procedural form of cell death with poor immunogenicity and physiology. ${ }^{4-7}$ Immunogenic apoptosis is unique in that it can elicit an immune response by exposing or releasing molecules, such as cytokines, chemokines, tumor-associated antigens (TAAs), and damageassociated molecular patterns (DAMPs), including exposure of calreticulin (CRT) at cell surface and release of high mobility group box 1 (HMGB1) and adenosine triphosphate (ATP). ${ }^{8,9}$ 
Sensitizers, such as photosensitizers or sonosensitizers, have recently been shown to destroy cancer cells by generating reactive oxygen species (ROS) in response to light or sound stimulation and by eliciting inflammatory responses during cell death. ${ }^{10}$ ICG has been identified as a possible sonosensitizer with minimum toxicity and has been approved by the United States Food and Drug Administration (FDA), exhibiting a low incidence of adverse side effects and can be administered directly for diagnostic purposes. ${ }^{11}$ Compared to free ICG, supramolecular systems such as nanoparticles have been demonstrated to significantly increase ROS production when exposed to exogenous energy sources. ${ }^{12} \mathrm{~A}$ primary immunostimulatory property of immunogenic apoptosis is that it enables the maturation of antigen-presenting cells (APCs), thereby eradicating dispersed or metastatic tumor cells through cytotoxic T lymphocytes (CTLs). ${ }^{13}$ However, because immunogenicity of TAAs and DAMPs can be outstandingly decreased during apoptosis progression, immunogenic apoptosis-inducing approaches have exhibited limited therapeutic responses, followed by proteolysis and intracellular oxidation to prevent autoimmunity. ${ }^{14-16}$

Necroptosis is a form of controlled and caspase-independent programmed cell death characterized by necrosis-like morphology, combining characters of necrosis and apoptosis, accompanied by cell membrane rupture, leakage of intracellular contents, and cytoplasmic swelling, activating the immune system and resulting in inflammation. 1 17, 18

Previous studies indicate that receptor-interacting protein kinase 3 (RIPK3) is critical in necroptosis to activate mixed lineage kinase domain-like protein (MLKL), thereby promoting cell membrane disruption. ${ }^{19,20}$ The immunogenicity of pro-inflammatory molecules (including DAMPs) is not significantly altered during necroptosis, since they are released without being exposed to severe surroundings that trigger oxidation and proteolysis, which is not the case during apoptosis. ${ }^{21}$

Consequently, necroptosis, one of three cell death mechanisms (necroptosis, apoptosis, and type 2 autophagic death), is one of the most effective approaches to boost antitumor immunity, implying its immense promise as a therapeutic target for cancer immunotherapy. ${ }^{22-25}$ However, broad implementations of necroptosis-inducing therapeutics in cancer therapy have been limited in clinics since RIPK3, and MLKL expression levels are downregulated in most cancer cells. ${ }^{26}$

SDT, which combines low-intensity ultrasound with sonosensitizers, has been investigated as a potential cancer treatment modality. ${ }^{11}$ Moreover, SDT demonstrates a high propensity for concentrating ultrasound energy to trigger sonosensitizers in far deeper tissue areas and to induce the sonosensitizer's local cytotoxicity when adequate sonosensitizer accumulation occurs at the tumor site. ${ }^{27,} 28$

Given that, we fabricated a novel multifunctional phase-transition nanoparticles platform system by constructing Lip-ICG-PFP-CRGD using liposome and indocyanine green (ICG) as the shell and perfluoropentane (PFP) as the core. The platform system described herein combined SDT with immunotherapy, inducing necroptosis with RIPK3/MLKL-independent for cancer treatment and eliciting cell membrane disruption through acoustic cavitation effect mediated by ultrasound. Triggered by 
ultrasound (US), the Lip-ICG-PFP-cRGD elicits ROS-mediated apoptosis. Moreover, the PFP phase change (from droplets to microbubbles) caused RIPK3/MLKL-independent necroptosis by acoustic cavitation effect, resulting in the release of biologically active DAMPs (CRT and HMGB1) to facilitate antitumor immunity (Scheme 1). In addition to their inherent contrasting ability under photoacoustic (PA) imaging, our liposomes may also be used as an imaging probe for US imaging after being irradiated with LIFU.

Such nanoparticles provided us with therapeutic and diagnostic vehicles to destroy ovarian cancer cells and improve antitumor efficacy when combined with SDT and immunotherapy.

\section{Materials And Methods}

\section{Materials}

Liposome includes 1,2-dipalmitoyl-sn-glycero-3-phosphocholine (DPPC), 1,2-distearoyl-sn-glycero-3phosphoethanolamine-N-[methoxy(polyethyleneglycol)-2000](DSPE-PEG2000), 1, 2-distearoyl-sn-glycero3-phospho-(1'-rac-glycerol) (DSPG) and cholesterol were purchased from Avanti Polar Lipids (Alabaster, AL, USA) Perfluoropentane (PFP), indocyanine green (ICG), propidium iodide (PI) and 1,10-dioctadecyl3,3,30,30-tetramethylindo-tricarbocyanine iodide (DiR) were obtained from Sigma Aldrich (St. Louis, MO, USA).

Reactive Oxygen Species Assay Kit (20,70-dichlorofluorescin diacetate, DCFH-DA), 1,10-dioctadecyl3,3,30,30-tetramethylindocarbocyanine perchlorate (Dil) were purchased from Santa Cruz Biotechnology (TX, USA). All reagents were of analytical grade and used without further purification.

\section{Preparation of LIP-ICG-PFP-CRGD, LIP-ICG-PFP}

The liposomes loaded with ICG and PFP, referred to as Lip-ICG-PFP-CRGD, were synthesized by a two-step emulsion method as described previously. ${ }^{29}$ Briefly, $5 \mathrm{mg}$ of DPPC, $2.0 \mathrm{mg}$ of DSPG, $1.5 \mathrm{mg}$ of DSPEPEG-CRGD, and $1.5 \mathrm{mg}$ of cholesterol were dissolved in $10 \mathrm{~mL}$ of methanol and $10 \mathrm{~mL}$ of chloroform. The solution was then transferred to a round bottom flask to form lipid films by rotary evaporation. After that, $2 \mathrm{~mL}$ of phosphate buffer saline (PBS) was added to the flask, sonicating the mixture to hydrate the lipid films for 5 min using ultrasound cleaner. Next, $0.05 \mathrm{~mL}$ of ICG aqueous solution $(10 \mathrm{mg} / \mathrm{mL})$ and $0.2 \mathrm{~mL}$ of PFP were added into lipid films and emulsified in an ice bath for 3 min using a sonicator (Heat System Inc, USA). Following that, a solution of LIP-ICG-PFP-cRGD was purified three times by centrifugation (8000 rpm, $5 \mathrm{~min}$ ), and the supernatant was removed. Finally, $2 \mathrm{~mL}$ of PBS were added to the precipitate for further experimentation. The LIP-ICG-PFP liposomes were synthesized by sonicating the hybrid of lipid films without CRGD and PFP in an ice bath for $3 \mathrm{~min}$ and then purified three times by centrifugation (8000 rpm, $5 \mathrm{~min})$.

\section{Characterization of liposome}


Nanoparticles (NPs) carrying PFP and ICG were successfully synthesized using DSPG, DSPE-PEG2000, DPPC, and cholesterol, and were called LIP-ICG-PFP-CRGD, generating a US-mediated cavitation effect. The diameter, zeta potential, and polydispersity index (PDI) of different kinds of NPs or NBs were measured by Zetasizer Nano ZS unit (Malvern Instruments, Malvern, UK) and their morphology and structure were observed by transmission electron microscopy (TEM). Ultraviolet-visible (UV-vis) spectrophotometer with scanning wavelength ranging from 200 to $900 \mathrm{~nm}$ was used to determine the absorption spectra of different nanoparticles. UV-vis spectrophotometer (260-Bio, Thermo Fisher Scientific) was used to evaluate the entrapment efficiency and loading of ICG, and entrapment efficiency and loading content were then calculated.

\section{PA and US Imaging In Vitro}

The agar-gel model (3\% agar w/v in double-distilled water) was utilized to evaluate LIP-ICG-PFP-CRGD capacity, which acts as contrast agents for dual-modal imaging. PA imaging of different concentrations of nanoparticles was shown on VEVO LASER PA imaging system (VEVO 2100, Canada).

For US imaging, liposomes were exposed to low-intensity focused ultrasound $(2 \sim 6 \mathrm{~W})$ for $100 \sim 300 \mathrm{~s}$. The contrast-enhanced ultrasound (CEUS) and B-mode images were obtained by an ultrasonic diagnostic instrument (MyLab 90; Esaote, Italy).

\section{Detection of ROS}

1, 3-Diphenylisobenzofuran (DPBF) was used to determine ROS generation in nanoparticles. A total of 60 $\mu \mathrm{L}$ of DPBF ( $4 \mathrm{mg} / \mathrm{mL}), 50 \mu \mathrm{L}$ of LIP-ICG-PFP-CRGD, and $1 \mathrm{~mL}$ of double distilled water were added into Eppendorf tube and exposed to low-intensity ultrasound with $2 \mathrm{~W} / \mathrm{cm}^{2}$ for 0-10 min in dark. Then, a multimode reader was used to evaluate ROS production by measuring the absorption at $410 \mathrm{~nm}$.

To detect intracellular ROS generation, 2', 7'-dichlorofluorescin diacetate (DCFH-DA) was employed as an indicator. In brief, 1 x $10^{5}$ ID8 cells were seeded in 12-well plates and co-incubated with LIP-ICG-PFP-CRGD (the concentration of each group was $0.4 \mathrm{mg} / \mathrm{mL}$ ) for $3 \mathrm{~h}$. All the wells were divided into six groups: control group, NPs group, only LIFU group ( $2 \mathrm{~W} / \mathrm{cm}^{2}$ for $200 \mathrm{~s}$ ), and NPs combined with LIFU group (2 $\mathrm{W} / \mathrm{cm}^{2}$ for $200 \mathrm{~s}$ ). Then, each of the wells was washed three times with PBS. Subsequently, the cells were cultured with diluted DCFH-DA for 30 min in dark. The ROS generation was evaluated by flow cytometry.

ID8 cells were seeded in confocal dishes at a density of $1 \times 10^{5}$ for $24 \mathrm{~h}$, and the rest of routine was as described above. Finally, laser scanning confocal microscopy (LSCM, Nikon AJR, Japan) was used to detect ROS generation.

\section{Determination of Cellular Uptake}

ID8 cells were seeded onto confocal dishes at a density of $1 \times 10^{5}$ for $24 \mathrm{~h}$. Next, the original media was replaced by media containing LIP-ICG-PFP or LIP-ICG-PFP-cRGD labeled with Dil dye for co-incubation 
with different periods $(1.0,2.0$, and $3.0 \mathrm{~h})$. Then, the cells were washed with PBS and cultured with 2-(4amidinophenyl)-6-indolecarbamidine dihydrochloride (DAPI) for 10 min to stain cell nuclei. Subsequently, PBS was used to wash the cells three times, and $4 \%$ paraformaldehyde was utilized to fix the cells.

Finally, the cellular uptake ability was evaluated by confocal laser scanning microscopy.

\section{In vitro cell viability}

To evaluate cytotoxicity in vitro, ID8 cells were seeded in a 96-well plate at a density of 5000 cells per well for $24 \mathrm{~h}$, and then, $100 \mu \mathrm{L}$ of LIP-ICG- PFP-CRGD nanoparticles with various concentrations were added and cultured for another $24 \mathrm{~h}$. Cell viabilities were tested by CCK-8 assay.

To test the therapeutic effects, ID8 cells were seeded in a 96-well plate and co-cultured with LIP-ICG-PFPCRGD nanoparticles (different concentrations were divided into five groups) for $3 \mathrm{~h}$. PBS was utilized to wash the wells three times, and the media in each well were displaced with fresh media. Next, the groups were exposed to low-intensity ultrasound with various power $\left(1.0,2.0,3.0,4.0\right.$, and $\left.5.0 \mathrm{w} / \mathrm{cm}^{2}\right)$ for 2 min in dark, and cell viabilities were determined using CCK-8 method.

\section{Cell death assay using annexin V/PI staining}

ID8 cells were cultured in 6-well dishes for $24 \mathrm{~h}$. Additionally, cells were incubated with liposomes and free ICG for $3 \mathrm{~h}$ and washed with PBS. Then, cells were trypsinized and re-suspended with $400 \mu \mathrm{L}$ of $1 \%$ FBS containing annexin $\mathrm{V}$ binding buffer. After that, the cells were exposed to LIFU for $300 \mathrm{~s}$ (power $6 \mathrm{~W}$, duty cycles: $50 \%$ ). The cells were further incubated for $1 \mathrm{~h}$ and stained with FITC annexin $\mathrm{V}$ and PI for 10 min. Finally, the cells were observed using a fluorescence microscope and analyzed with flow cytometry.

\section{The CRT expression and HMGB1 Release Assay}

The ID8 cells were co-cultured with nanoparticles (LIP-ICG-PFP-cRGD $0.4 \mathrm{mg} / \mathrm{mL}$ ) in 6-well plates for $3 \mathrm{~h}$. All the wells were divided into four groups: control group, only LIFU group, only NPs group, and LIP-ICGPFP-cRGD plus LIFU group ( $4 \mathrm{~W} / \mathrm{cm}^{2}$ for $200 \mathrm{~s}$ ). The cells were cultured for another $18 \mathrm{~h}$, and the supernatant was added into another 6-well plate which was cultured with dendritic cells (DC) for $6 \mathrm{~h}$. Subsequently, the cells and supernatants from DC co-cultured were collected to examine the CRT and HMGB1 level by ELISA (LifeSpan BioSciences), according to manufacturer's instructions.

\section{The expression of CD86 in dendritic cells}

To test the expression of CD86 (a marker for DC maturation), ID8 cells were seeded and co-incubated with different nanoparticles. Following exposure to different treatments, the supernatant was collected separately and added into another 12-well plate cultured with DC for $24 \mathrm{~h}$. After washing with PBS and staining with FITC-conjugated anti-CD86 for 60 min in dark, the samples were analyzed using flow cytometry to quantify CD86 expression in DC.

\section{Statistical analysis}


The experimental data were analyzed using GraphPad Prism (version 8.0) with the unpaired Student's ttest, or the paired Student's t-test. P-values $<0.05$ indicated statistically significant difference $\left({ }^{*} p<0.05\right.$, $\star \star p<0.01)$.

\section{Results And Discussion}

\section{Characterization}

Previous studies developed a robust stealthy phospholipid-liposome that

showed long-circulation and could be stimulated by NIR light to release encapsulated drugs. ${ }^{30}$ Compared to NIR irradiation, US achieves much deeper penetration, and its energy can be regulated in exposed areas.

The liposomes were synthesized using a two-step emulsion method. ${ }^{31}$ Transmission electron microscopy demonstrated a nearly spherical structure of lip-ICG-PFP and Lip-ICG-PFP-cRGD with smooth surfaces (Figs. 1A and B). Light scattering measurements manifested a mean diameter of $300 \mathrm{~nm}$ (PDI 0.135) with PFP incorporation, indicating uniform particle size distributions (Fig. 1D). Zeta potentials of lip-ICGPFP and lip-ICG-PFP-CRGD were $21.47 \pm 0.59 \mathrm{mV}$ and $21.47 \pm 0.59 \mathrm{mV}$ (Fig. 1C) at pH = 7.4, respectively. Furthermore, Lip-ICG-PFP-cRGD could maintain appreciable stability in cell culture medium at $4{ }^{\circ} \mathrm{C}$ for 30 days (Fig. 1E) without significant diameter changes, suggesting excellent long-term stability.

The UV-vis absorption spectra (Fig. 2A) of Lip-ICG-PFP and Lip-ICG-PFP-CRGD display prominent absorption peaks of ICG at $790 \mathrm{~nm}$, indicating that ICG had successful encapsulation into nanoparticles. To calculate the encapsulation efficiency of ICG in liposomes, a standard curve was drawn (Fig. 2B), and ICG amount encapsulated in Lip-ICG-PFP-CRGD was determined spectrophotometrically as a $92 \%$ loading efficiency. The individual structure of Lip-ICG-PFP-cRGD was discovered to encapsulate vaporable PFP, which generated nanobubbles and fused into microbubbles after triggering by LIFU. The PFP encapsulated inside the liposome was converted into numerous microbubbles within 2 min when treated with LIFU (Fig. 2D) and was visualized using optical microscopy. As the power increased, nanoparticles with PFP cores could be converted into microbubbles. The adjacent microbubbles could fuse with each other to form larger microbubbles until they burst, consistent with previous studies, revealing the excellent ability to function as a US imaging agent and acoustic cavitation eliciting necroptosis.

\section{In Vitro PA and Ultrasound Imaging of Lip-ICG-PFP-CRGD}

The individual structure of liposome was creatively discovered to encapsulate vaporable PFP, which generated nanobubbles and fused into microbubbles after triggering by LIFU, thus realizing the enhanced US imaging. Thus, it was interesting to investigate the echogenic property of the nanoparticles with US irradiation in vitro. We speculated that phase-changeable liposomes could serve as ultrasound agents to enhance US imaging. US images of liposome showed acoustic reflectivity contrast contributing to the generation of gas bubbling under US irradiation, consistent with quantitative production results (Fig. 3A, B 
and C). In this regard, enhanced US imaging showed that Lip-ICG-PFP-CRGD was considered an ultrasound contrast agent. The ICG can act as a PA agent. To explore the potential of nanoparticles as a PA probe, PA imaging was acquired using Lip-ICG-PFP-CRGD as the contrast agent in vitro. With the excitation wavelength ranging from $680 \mathrm{~nm}$ to $950 \mathrm{~nm}$, liposome had the strongest PA signal at 700 to $850 \mathrm{~nm}$ (Fig. 3E). As shown in Fig. 3D, PA images of Lip-ICG-PFP-CRGD dispersed in aqueous solution at different concentrations clearly indicate their contrast-enhancement performances under excitation at $800 \mathrm{~nm}$.

\section{Targeting Efficiency in Vitro}

The efficacy of Dil-labeled liposomes to target ID8 cells was verified by CLSM. As presented in Fig. 4A, the fluorescence intensity was almost equally weak in ID8 cancer cells after $1 \mathrm{~h}$ of incubation with Lip-ICGPFP and Lip-ICG-PFP-CRGD, and a higher level of cellular uptake could be observed when the incubation time increased from 2 to $3 \mathrm{~h}$. Considerable fluorescence intensity in ID8 cells incubated with Lip-ICG-PFPCRGD for $3 \mathrm{~h}$ was observed, whereas a much weaker fluorescent signal was exhibited in ID8 cells incubated with Lip-ICG-PFP. This result corresponds to the quantitative analysis (Fig. 4B), indicating that cRGD can lead to a higher cell internalization of drugs.

\section{ROS Generation}

Given that ICG is one of the promising forms of sonosensitizers, DPBF was applied to detect ROS production. As the absorbance intensity of DPBF at $410 \mathrm{~nm}$ in UV-vis spectrum decreases when it is oxidized by ROS, DPBF consumption assists in calculating ROS production. As displayed in Fig. 2C, DPBF consumption in liposome plus LIFU group was notably higher compared to that in control group, and with prolonged irradiation time, the liposomes exhibited obviously higher ROS production efficacy, indicating its potential as sonosensitizer for SDT.

To reveal the intracellular mechanism of Lip-ICG-PFP-cRGD as sonosensitizers in killing cancer cells, intracellular ROS levels were determined using DCFH-DA, which could be converted from nonfluorescence status into fluorescent 2,7-dichlorofluorescein (DCF) by ROS. It can be found that Lip-ICGPFP-CRGD combined with LIFU irradiation induces mass production of intracellular ROS as demonstrated by strong green fluorescence in cancer cells (Figs. 5A and B). Comparatively, neither control cells nor US irradiation only could induce the intracellular fluorescence. It can be deduced that nanoparticles as sonosensitizers can generate ROS under LIFU irradiation to induce the toxic effect and achieve the therapeutic function afterward.

\section{Antitumor Effect in Vitro and ICD-induced Immune Priming}

The toxicity of Lip-ICG-PFH-cRGD was measured by CCK-8 assay after co-incubation with ID8 cells for 24 $\mathrm{h}$ at different concentrations. As Fig. 6A displays, after $24 \mathrm{~h}$ of incubation, Lip-ICG-PFP-cRGD revealed no significant cytotoxicity on ID8 cells at a concentration up to $1000 \mu \mathrm{g} / \mathrm{mL}$, indicating well in vitro cytocompatibility. No apparent cytotoxicity occurs within the therapeutic concentration range employed 
in this study. Next, to observe SDT effectiveness of liposomes, ID8 cells treated with various concentration of nanoparticles were irradiated with LIFU for different time range. As expected, the cell viability decreased as the concentration and irradiation time increased, implying a dose-dependent cytotoxic effect. And more than $60 \%$ of cells were killed by cytotoxic ROS and acoustic cavitation, eliciting necroptosis in the presence of LIFU. (Figs. 6B and C).

Necroptosis is distinct from apoptosis in morphological features such as cell membrane rupture and cytoplasmic swelling. By comparison, apoptosis is characterized by cell shrinkage, nuclear fragmentation, and formation of apoptotic bodies. ${ }^{32}$ To evaluate the cell death mechanism, the annexin $\mathrm{V} /$ propidium iodide $(\mathrm{PI}$ ) assay was carried out by treating the samples with ID8 cancer cells (Figs. 6D and E). ${ }^{33}$ When the cells were exposed to liposome or LIFU only, no significant morphology changes of cellular membrane were observed without fluorescent signals from annexin $\mathrm{V}$ or PI, suggesting that LIFU irradiation and nanoparticles did not cause apparent damage to the cells. However, for the free ICG plus LIFU treated cells, apoptotic features (i.e., chromatin condensation of cells and externalization of phosphatidylserine) were clearly shown. Interestingly, Lip-ICG-PFP-cRGD treated cells exhibited a loss in their cell morphology under LIFU irradiation, suggesting that the cell membrane was damaged by USmediated intracellular explosion of Lip-ICG-PFP-cRGD. The disintegration of cell membrane by Lip-ICGPFP-CRGD treatment led to leakage of chromatins, cytosolic components, and membrane fragments. ${ }^{34}$ These results implied that, upon LIFU irradiation, Lip-ICG-PFP-CRGD could induce necroptosis of the cells by physical disruption of the cell membrane. Interestingly, regardless of RIPK3 and MLKL expression, the cells exhibited morphological features of necroptosis when they were treated with Lip-ICG-PFP-CRGD under LIFU irradiation, implying that necroptosis of cells occurred in a RIPK3/MLKL-independent manner.

In contrast to apoptosis, necroptosis provokes the release of intact DAMPs from the cells, thus effectively mediating the maturation and activation of dendritic cells (DCs). Of the DAMPs released, HMGB1 has often been considered a necroptosis representative marker. ${ }^{21}$ As depicted in Fig. 7A, Lip-ICG-PFP-CRGD plus LIFU could induce a significant extracellular release of HMGB1. Additionally, immunogenic cell death (ICD) of tumor cells is characterized by eliciting cell surface expression of pro-apoptotic calreticulin (CRT). ${ }^{9}$ The exposure of CRT on cancer cell surface serves as an "eat me" signal to antigen presentation cells (e.g., DCs and macrophages) and elicits antitumor immune responses. After treatment of Lip-ICGPFP-CRGD plus LIFU, CRT exposure on ID8 tumor cells was significantly elevated (Fig. 7B). To further evaluate the efficacy of turning tumor cells into antigen-presenting cells, DC maturation was investigated. As a result, CD86 expression, a DC maturation marker, remarkably increased via Lip-ICG-PFP-CRGD treatment upon LIFU irradiation compared with other control groups (Fig. 7C). Overall, it was evident that Lip-ICG-PFP-cRGD effectively provoked LIFU-mediated necroptosis in a RIPK3/MLKL-independent manner, resulting in releasing intact DAMPs for enhancing the antitumor immunity.

\section{Conclusions}


In summary, we demonstrate that nanoparticles can trigger necroptosis in ovarian cancer cells expressing low RIPK3, which ruptures cell-membrane by acoustic cavitation effect. The nanoparticles were loaded with PFP and ICG to enhance antitumor efficacy and immunological effects. In addition, the liposome was demonstrated to be a bimodal imaging probe for PA/US imaging. When exposed to low-intensity focused ultrasound, the nanoparticles effectively facilitate the release of damage-associated molecular patterns through burst-mediated cell-membrane decomposition. Therefore, necroptosis-inducible nanoparticles remarkably enhance antitumor immunity by activating $\mathrm{CD} 8^{+}$cytotoxic $T$ cells and maturing dendritic cells in vitro. In summary, the combination of SDT and immunotherapy can significantly improve anti-cancer efficacy and overcome drug resistance through nanoparticles, a promising approach for ovarian cancer therapy.

\section{Declarations}

\section{Ethics approval and consent to participate}

Not applicable.

\section{Consent for publication}

Not applicable.

\section{Availability of data and material}

All data generated or analyzed during this study are included in this published article.

\section{Competing interests}

The authors declare that they have no competing interests.

\section{Funding}

This work was supported by the National Natural Science Foundation of China (81630047, 82071926), the Science and Technology Research Program of Chongqing Municipal Education Commission (Grant No. KJZD-K202000402).

\section{Authors' contributions}

WZ and HTR planed and designed the experiments and drafted the main part of the manuscript. RHT offered the fund to perform this study. MML, YZ, MY, and YC performed the in vitro study and analyzed the data. All authors read and approved the final manuscript.

\section{Acknowledgements}

Not applicable. 


\section{References}

1. Siegel, R. L.; Miller, K. D.; Fuchs, H. E.; Jemal, A., Cancer Statistics, 2021. CA: a cancer journal for clinicians 2021, 71 (1), 7-33.

2. Torre, L. A.; Bray, F.; Siegel, R. L.; Ferlay, J.; Lortet-Tieulent, J.; Jemal, A., Global cancer statistics, 2012. CA: a cancer journal for clinicians 2015, 65 (2), 87-108.

3. Srivastava, S. K.; Ahmad, A.; Miree, O.; Patel, G. K.; Singh, S.; Rocconi, R. P.; Singh, A. P., Racial health disparities in ovarian cancer: not just black and white. Journal of ovarian research 2017, 10 (1), 58.

4. Erwig, L. P.; Henson, P. M., Clearance of apoptotic cells by phagocytes. Cell death and differentiation 2008, 15 (2), 243-50.

5. Green, D. R.; Ferguson, T.; Zitvogel, L.; Kroemer, G., Immunogenic and tolerogenic cell death. Nature reviews. Immunology 2009, 9 (5), 353-63.

6. Tesniere, A.; Panaretakis, T.; Kepp, O.; Apetoh, L.; Ghiringhelli, F.; Zitvogel, L.; Kroemer, G., Molecular characteristics of immunogenic cancer cell death. Cell death and differentiation 2008, 15 (1), 3-12.

7. Morioka, S.; Maueröder, C.; Ravichandran, K. S., Living on the Edge: Efferocytosis at the Interface of Homeostasis and Pathology. Immunity 2019, 50 (5), 1149-1162.

8. Montico, B.; Nigro, A.; Casolaro, V.; Dal Col, J., Immunogenic Apoptosis as a Novel Tool for Anticancer Vaccine Development. International journal of molecular sciences 2018, 19 (2).

9. Galluzzi, L.; Buqué, A.; Kepp, O.; Zitvogel, L.; Kroemer, G., Immunogenic cell death in cancer and infectious disease. Nat Rev Immunol 2017, 17(2), 97-111.

10. Sang, W.; Zhang, Z.; Dai, Y.; Chen, X., Recent advances in nanomaterial-based synergistic combination cancer immunotherapy. Chemical Society reviews 2019, 48 (14), 3771-3810.

11. Son, S.; Kim, J. H.; Wang, X.; Zhang, C.; Yoon, S. A.; Shin, J.; Sharma, A.; Lee, M. H.; Cheng, L.; Wu, J.; Kim, J. S., Multifunctional sonosensitizers in sonodynamic cancer therapy. Chemical Society reviews 2020, 49 (11), 3244-3261.

12. Lin, H.; Li, S.; Wang, J.; Chu, C.; Zhang, Y.; Pang, X.; Lv, P.; Wang, X.; Zhao, Q.; Chen, J.; Chen, H.; Liu, W.; Chen, X.; Liu, G., A single-step multi-level supramolecular system for cancer sonotheranostics. Nanoscale horizons 2019, 4 (1), 190-195.

13. Duan, X.; Chan, C.; Lin, W., Nanoparticle-Mediated Immunogenic Cell Death Enables and Potentiates Cancer Immunotherapy. Angewandte Chemie (International ed. in English) 2019, 58 (3), 670-680.

14. Sachet, M.; Liang, Y. Y.; Oehler, R., The immune response to secondary necrotic cells. Apoptosis : an international journal on programmed cell death 2017, 22 (10), 1189-1204.

15. Strasser, A.; Jost, P. J.; Nagata, S., The many roles of FAS receptor signaling in the immune system. Immunity 2009, 30 (2), 180-92.

16. Kazama, H.; Ricci, J. E.; Herndon, J. M.; Hoppe, G.; Green, D. R.; Ferguson, T. A., Induction of immunological tolerance by apoptotic cells requires caspase-dependent oxidation of high-mobility group box-1 protein. Immunity 2008, 29 (1), 21-32. 
17. Um, W.; Ko, H.; You, D. G.; Lim, S.; Kwak, G.; Shim, M. K.; Yang, S.; Lee, J.; Song, Y.; Kim, K.; Park, J. H., Necroptosis-Inducible Polymeric Nanobubbles for Enhanced Cancer Sonoimmunotherapy. Adv Mater 2020, 32 (16), e1907953.

18. Zhang, Y. Y.; Liu, H., Connections between various trigger factors and the RIP1/ RIP3 signaling pathway involved in necroptosis. Asian Pacific journal of cancer prevention : APJCP 2013, 14 (12), 7069-74.

19. Zhang, D. W.; Shao, J.; Lin, J.; Zhang, N.; Lu, B. J.; Lin, S. C.; Dong, M. Q.; Han, J., RIP3, an energy metabolism regulator that switches TNF-induced cell death from apoptosis to necrosis. Science (New York, N.Y.) 2009, 325 (5938), 332-6.

20. Zhao, J.; Jitkaew, S.; Cai, Z.; Choksi, S.; Li, Q.; Luo, J.; Liu, Z. G., Mixed lineage kinase domain-like is a key receptor interacting protein 3 downstream component of TNF-induced necrosis. Proceedings of the National Academy of Sciences of the United States of America 2012, 109 (14), 5322-7.

21. Kaczmarek, A.; Vandenabeele, P.; Krysko, D. V., Necroptosis: the release of damage-associated molecular patterns and its physiological relevance. Immunity 2013, 38 (2), 209-23.

22. Degterev, A.; Huang, Z.; Boyce, M.; Li, Y.; Jagtap, P.; Mizushima, N.; Cuny, G. D.; Mitchison, T. J.; Moskowitz, M. A.; Yuan, J., Chemical inhibitor of nonapoptotic cell death with therapeutic potential for ischemic brain injury. Nat Chem Biol 2005, 1 (2), 112-9.

23. Aaes, T. L.; Kaczmarek, A.; Delvaeye, T.; De Craene, B.; De Koker, S.; Heyndrickx, L.; Delrue, I.; Taminau, J.; Wiernicki, B.; De Groote, P.; Garg, A. D.; Leybaert, L.; Grooten, J.; Bertrand, M. J.; Agostinis, P.; Berx, G.; Declercq, W.; Vandenabeele, P.; Krysko, D. V., Vaccination with Necroptotic Cancer Cells Induces Efficient Anti-tumor Immunity. Cell reports 2016, 15 (2), 274-87.

24. Pasparakis, M.; Vandenabeele, P., Necroptosis and its role in inflammation. Nature 2015, 517(7534), 311-20.

25. Suntharalingam, K.; Awuah, S. G.; Bruno, P. M.; Johnstone, T. C.; Wang, F.; Lin, W.; Zheng, Y. R.; Page, J. E.; Hemann, M. T.; Lippard, S. J., Necroptosis-inducing rhenium(V) oxo complexes. Journal of the American Chemical Society 2015, $137(8), 2967-74$.

26. Koo, G. B.; Morgan, M. J.; Lee, D. G.; Kim, W. J.; Yoon, J. H.; Koo, J. S.; Kim, S. I.; Kim, S. J.; Son, M. K.; Hong, S. S.; Levy, J. M.; Pollyea, D. A.; Jordan, C. T.; Yan, P.; Frankhouser, D.; Nicolet, D.; Maharry, K.; Marcucci, G.; Choi, K. S.; Cho, H.; Thorburn, A.; Kim, Y. S., Methylation-dependent loss of RIP3 expression in cancer represses programmed necrosis in response to chemotherapeutics. Cell research 2015, 25 (6), 707-25.

27. Chen, Y. W.; Liu, T. Y.; Chang, P. H.; Hsu, P. H.; Liu, H. L.; Lin, H. C.; Chen, S. Y., A theranostic nrGO@MSN-ION nanocarrier developed to enhance the combination effect of sonodynamic therapy and ultrasound hyperthermia for treating tumor. Nanoscale 2016, 8 (25), 12648-57.

28. Qian, X.; Zheng, Y.; Chen, Y., Micro/Nanoparticle-Augmented Sonodynamic Therapy (SDT): Breaking the Depth Shallow of Photoactivation. Adv Mater 2016, 28 (37), 8097-8129.

29. Jian, J.; Liu, C.; Gong, Y.; Su, L.; Zhang, B.; Wang, Z.; Wang, D.; Zhou, Y.; Xu, F.; Li, P.; Zheng, Y.; Song, L.; Zhou, X., India ink incorporated multifunctional phase-transition nanodroplets for 
photoacoustic/ultrasound dual-modality imaging and photoacoustic effect based tumor therapy. Theranostics 2014, 4 (10), 1026-38.

30. Ge, R.; Cao, J.; Chi, J.; Han, S.; Liang, Y.; Xu, L.; Liang, M.; Sun, Y., NIR-guided dendritic nanoplatform for improving antitumor efficacy by combining chemo-phototherapy. International journal of nanomedicine 2019, 14, 4931-4947.

31. Zhao, X. Z.; Zhang, W.; Cao, Y.; Huang, S. S.; Li, Y. Z.; Guo, D.; Wang, X. Y.; Ran, H. T., A Cleverly Designed Novel Lipid Nanosystem: Targeted Retention, Controlled Visual Drug Release, and Cascade Amplification Therapy for Mammary Carcinoma in vitro. Int J Nanomedicine 2020, 15, 3953-3964.

32. Vanden Berghe, T.; Vanlangenakker, N.; Parthoens, E.; Deckers, W.; Devos, M.; Festjens, N.; Guerin, C.; Brunk, U.; Declercq, W.; Vandenabeele, P., Necroptosis, necrosis and secondary necrosis converge on similar cellular disintegration features. Cell death and differentiation 2010, 17 (6), 922-30.

33. Yang, H.; Ma, Y.; Chen, G.; Zhou, H.; Yamazaki, T.; Klein, C.; Pietrocola, F.; Vacchelli, E.; Souquere, S.; Sauvat, A.; Zitvogel, L.; Kepp, O.; Kroemer, G., Contribution of RIP3 and MLKL to immunogenic cell death signaling in cancer chemotherapy. Oncoimmunology 2016, 5 (6), e1149673.

34. Gong, Y.; Crawford, J.; Heckmann, B.; Green, D., To the edge of cell death and back. The FEBS journal 2019, 286 (3), 430-440.

\section{Figures}




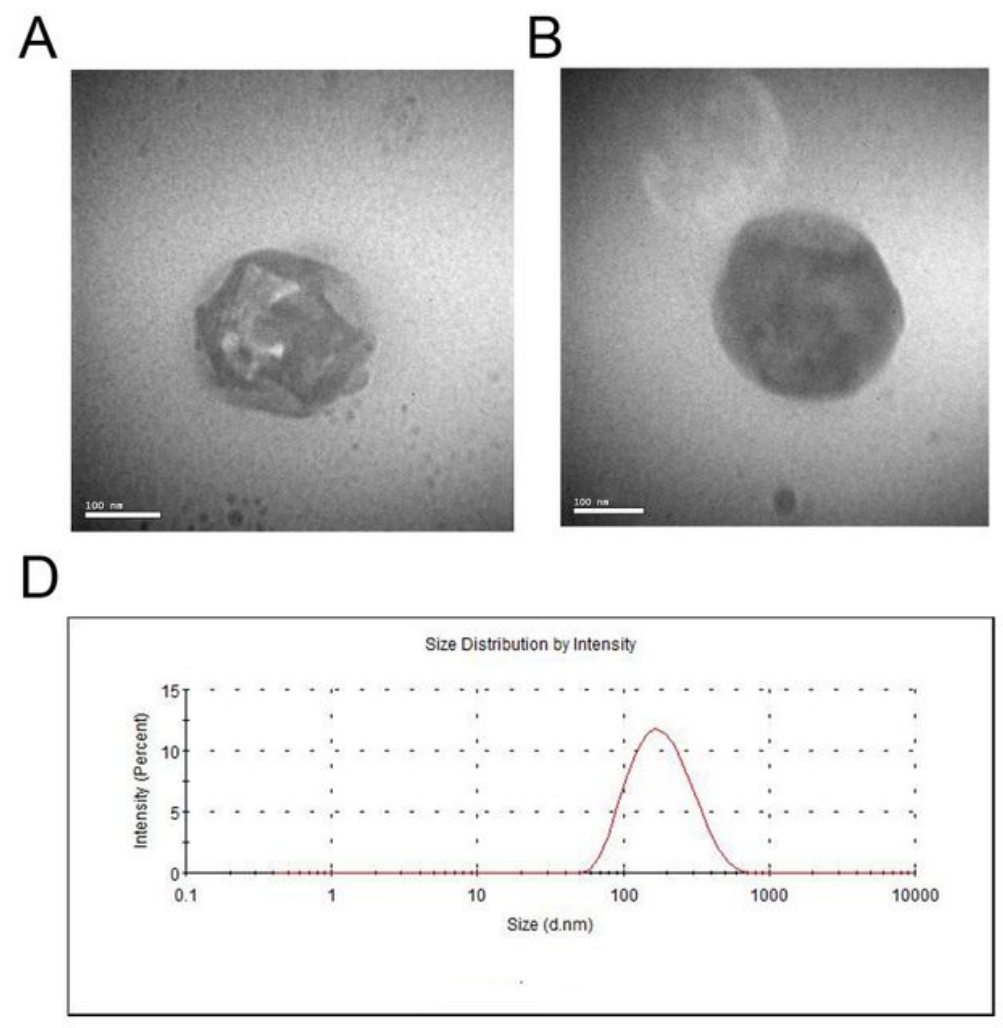

\section{C}

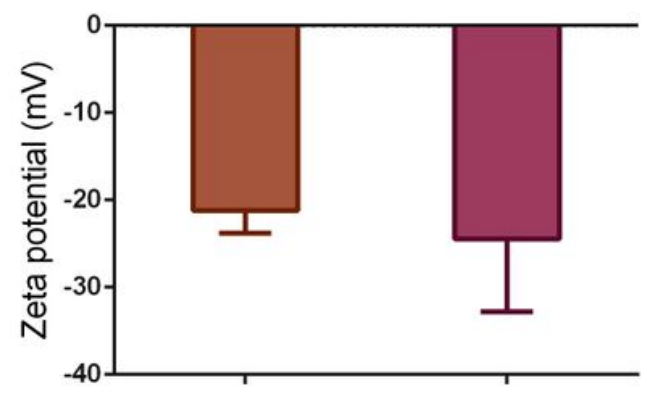

E

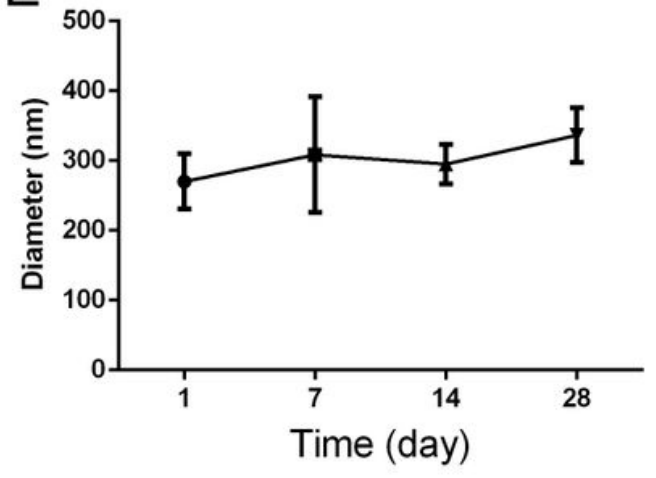

Figure 1

Characterization of nanoparticles. (A, B) TEM images of Lip-ICG-PFP and Lip-ICG-PFP-cRGD. (C) Zeta potential of the Lip-ICG-PFP and Lip-ICG-PFP-CRGD. (D) Size distribution of Lip-ICG-PFP-CRGD. (E) Size changes of Lip-ICG-PFP-CRGD at $4{ }^{\circ} \mathrm{C}$ for 30 days $(n=3)$. 


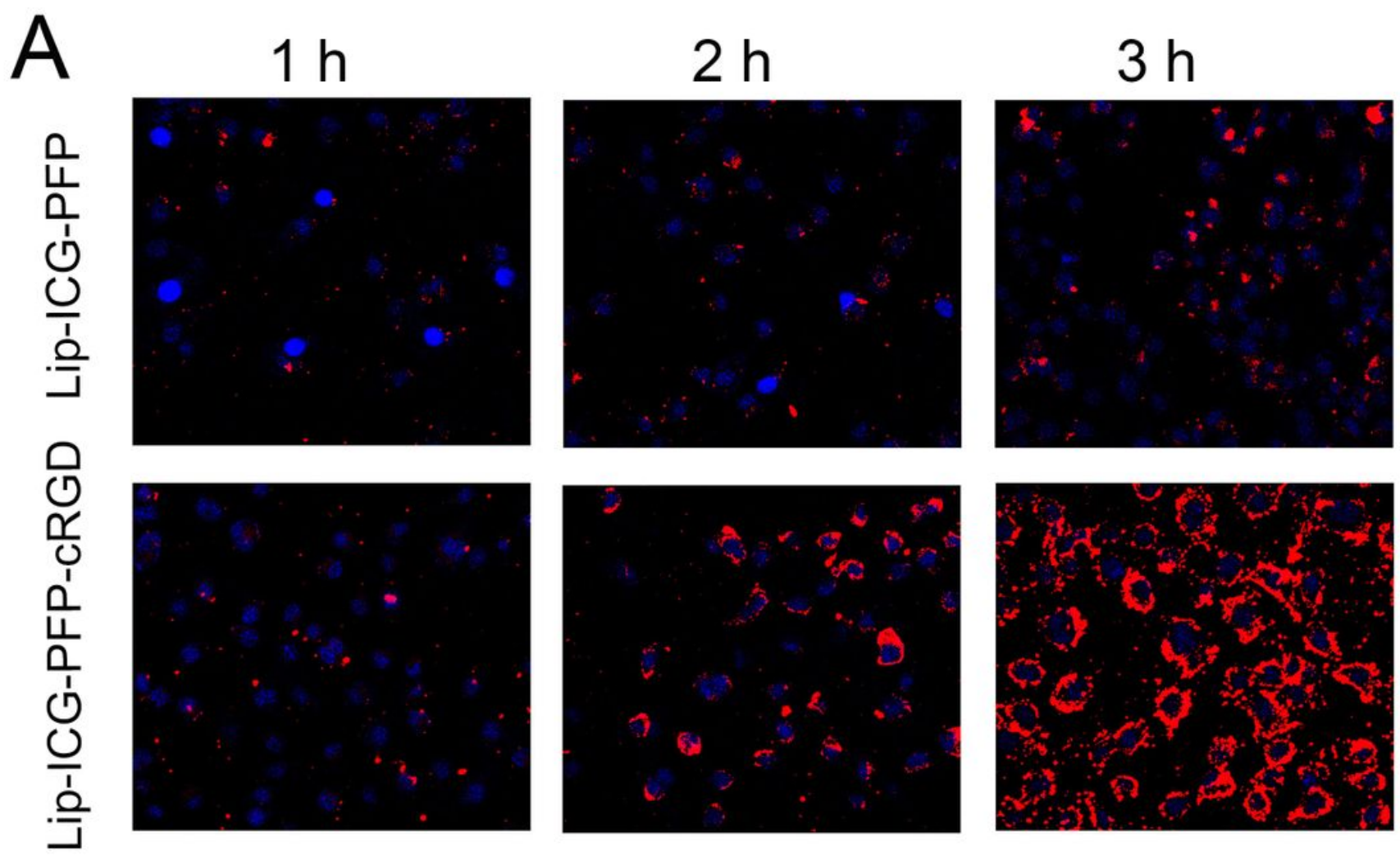

B

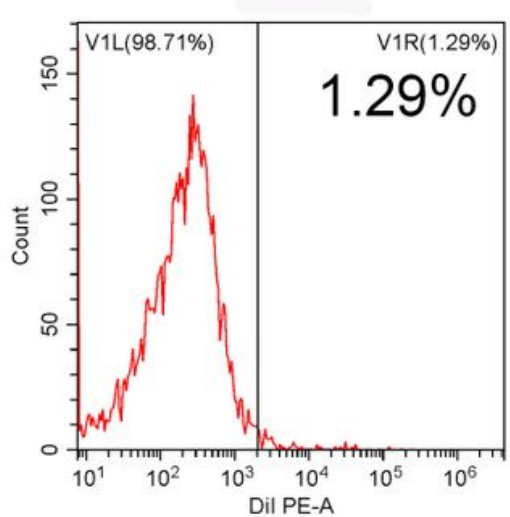

Control

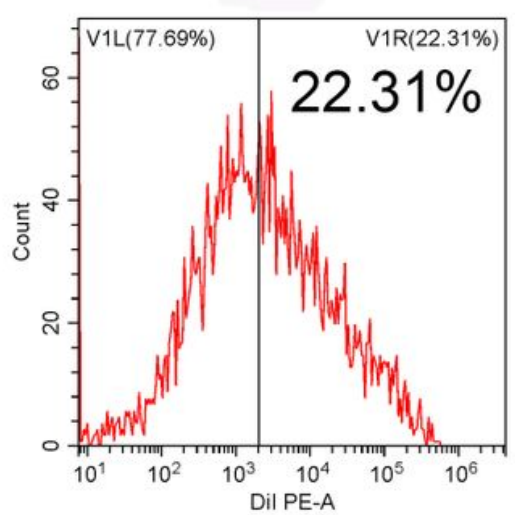

Lip-ICG-PFP

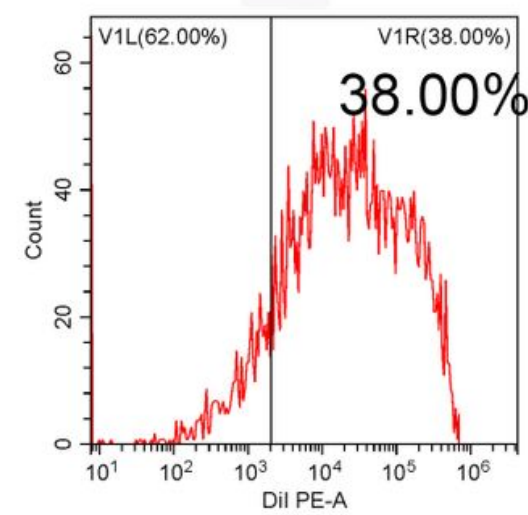

Lip-ICG-PFP-cRGD

Figure 2

(A) Identification of liposomes by UV-vis spectroscopy. (B) The concentration-absorbance standard curve of ICG. (C) DPBF consumption of liposomes under LIFU irradiation. (D) Optical images of Lip-ICG-PFPCRGD after LIFU irradiation (2 min, 2-6 W). 
A
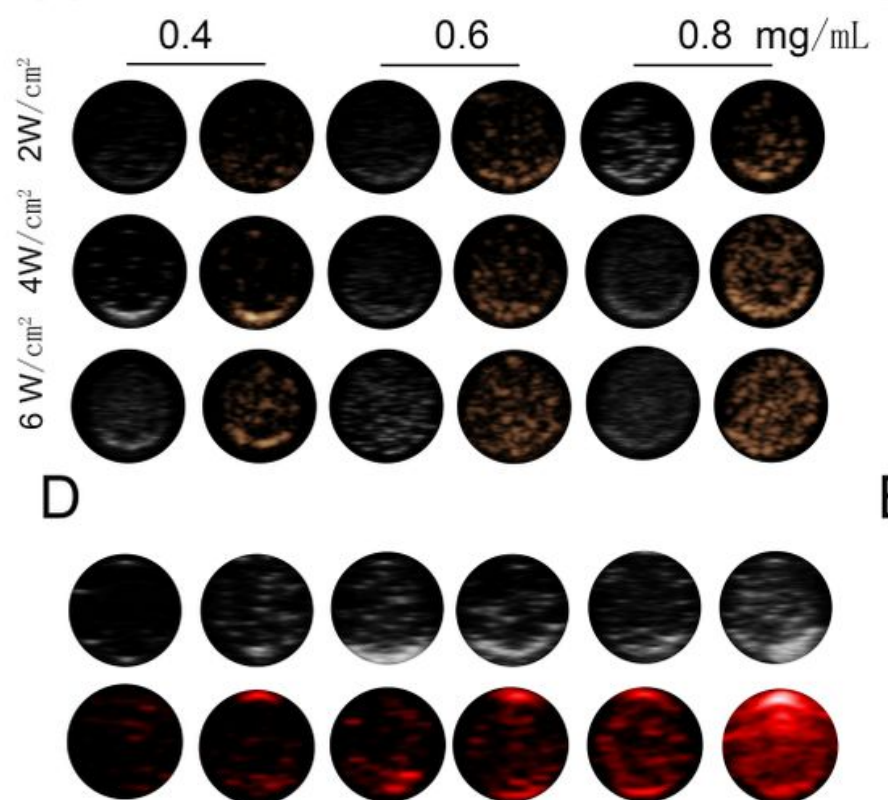

0.2

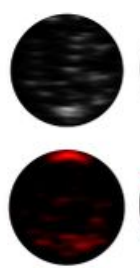

0.4

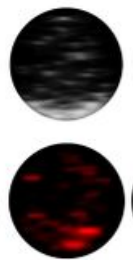

0.6

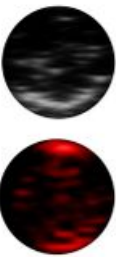

0.8

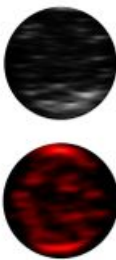

1.0

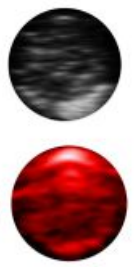

$2.0 \mathrm{mg} / \mathrm{mL}$

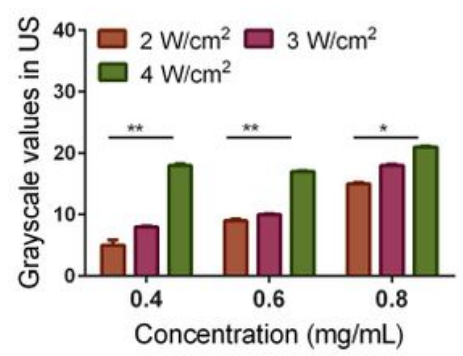

E

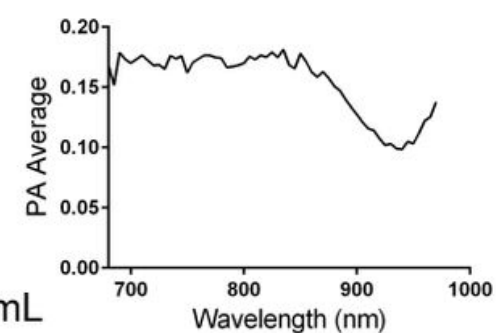

C

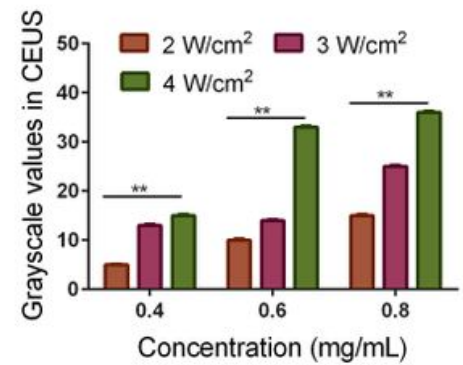

F

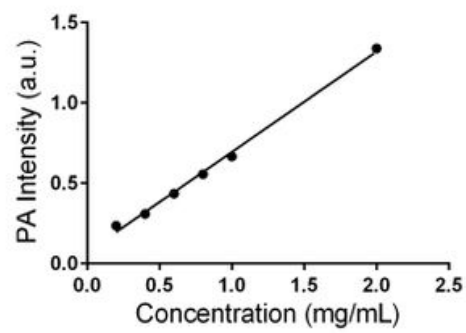

Figure 3

(A) B-mode and CEUS images of Lip-ICG-PFP-CRGD after LIFU irradiation under different conditions (2-4 $\mathrm{W} / \mathrm{cm} 2,1 \mathrm{~min}$ ). (B, C) The grayscale value in B-mode and CEUS after irradiated with LIFU (the data are shown as mean $\pm S D, n=3$ ). (D) PA images of Lip-ICG-PFP-cRGD with different concentration gradient. (E) Quantitative PA intensities of liposome in vitro. (F) Plot of PA values of Lip-ICG-PFP-CRGD with various concentration gradient. 


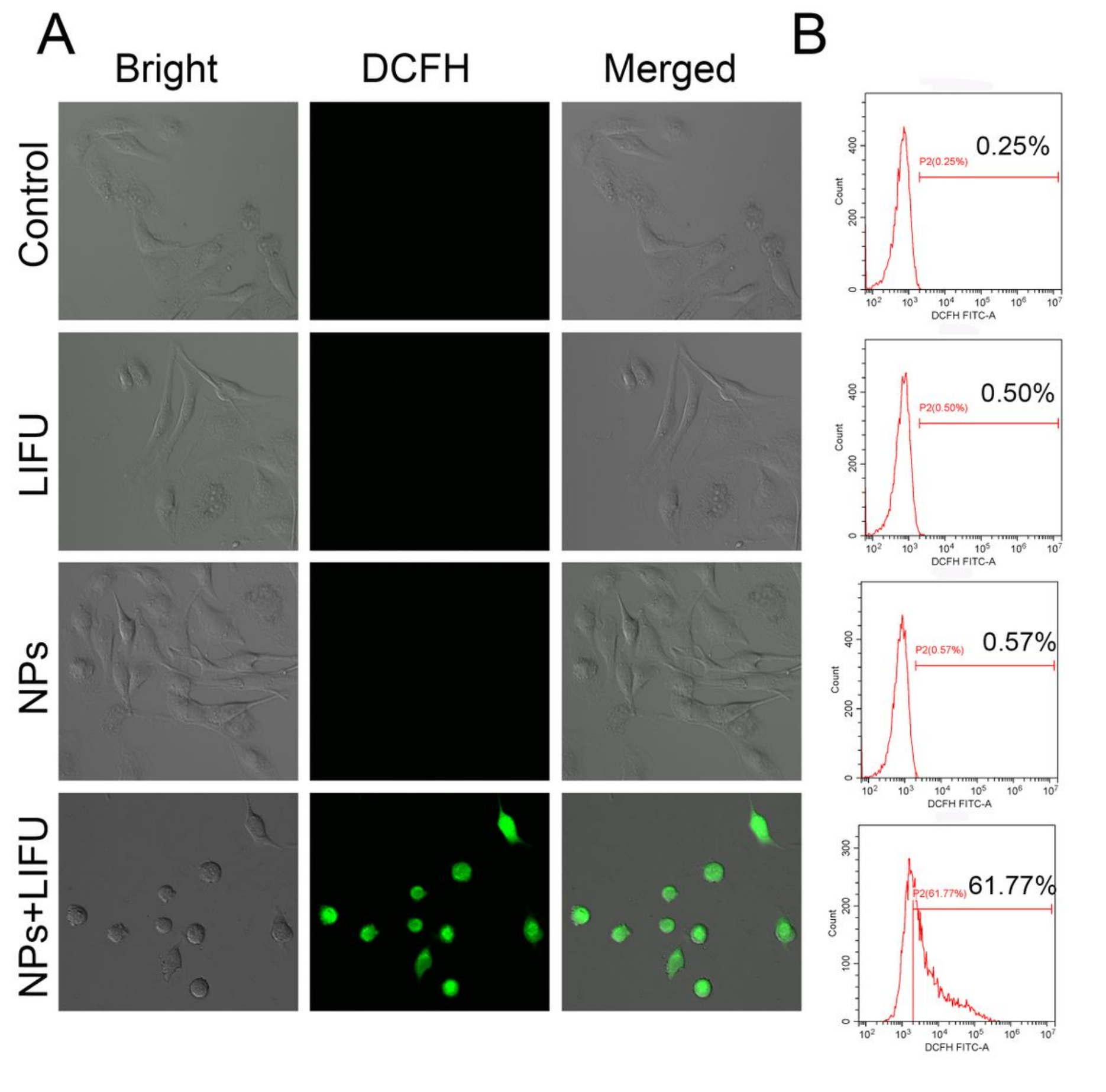

Figure 4

Targeting efficiency of liposomes in vitro. (A) CLSM images of ID8 cells after incubation with Lip-ICGPFP-cRGD and Lip-ICG-PFP nanoparticles for elevated time. (B) Flow cytometry results of ID8 cells after incubation with Lip-ICG-PFP-cRGD and Lip-ICG-PFP nanoparticles for $2 \mathrm{~h}$. 
A

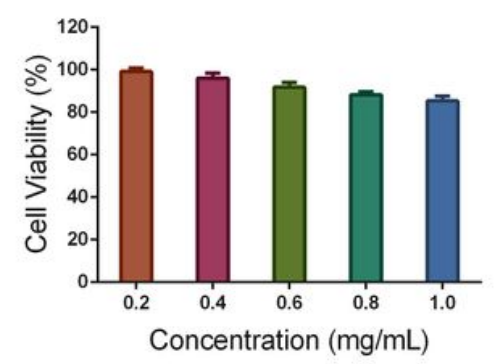

D
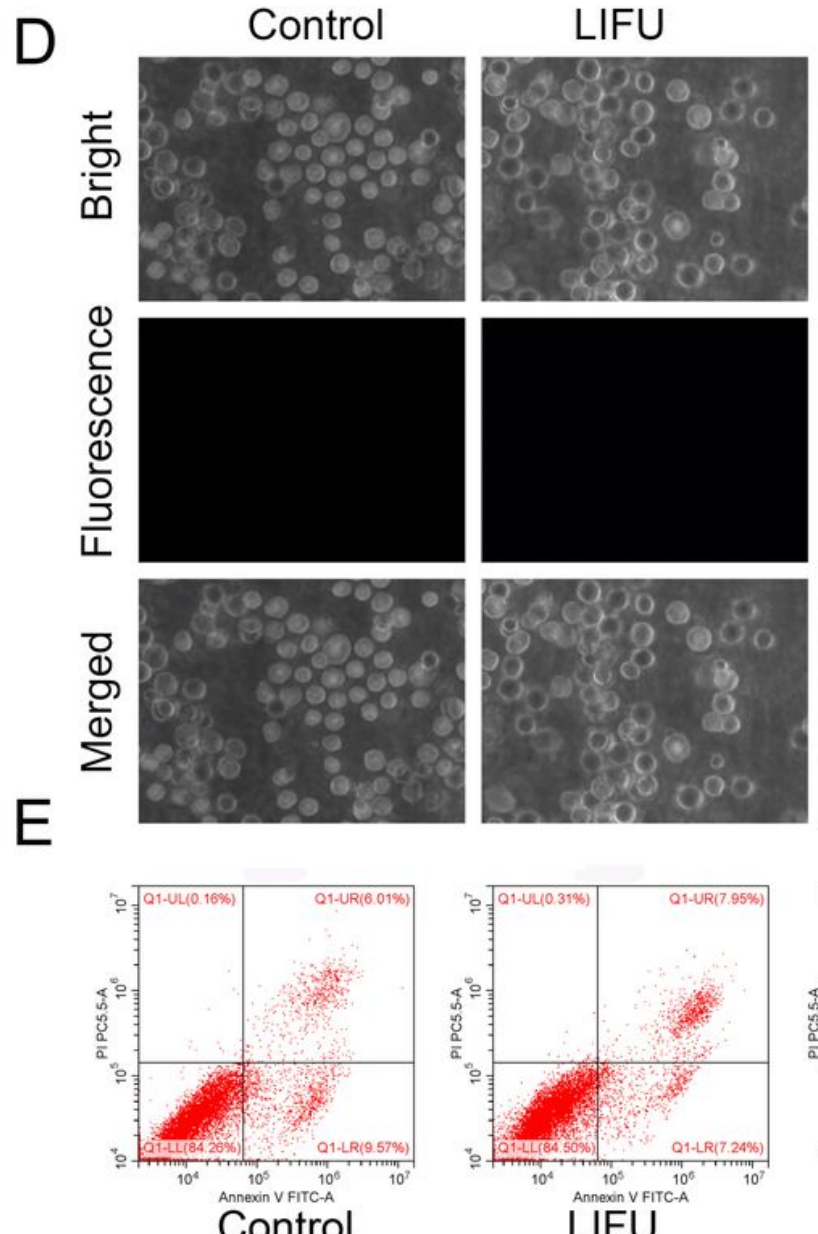

Control

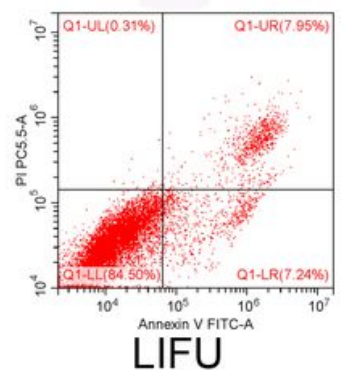

LIFU
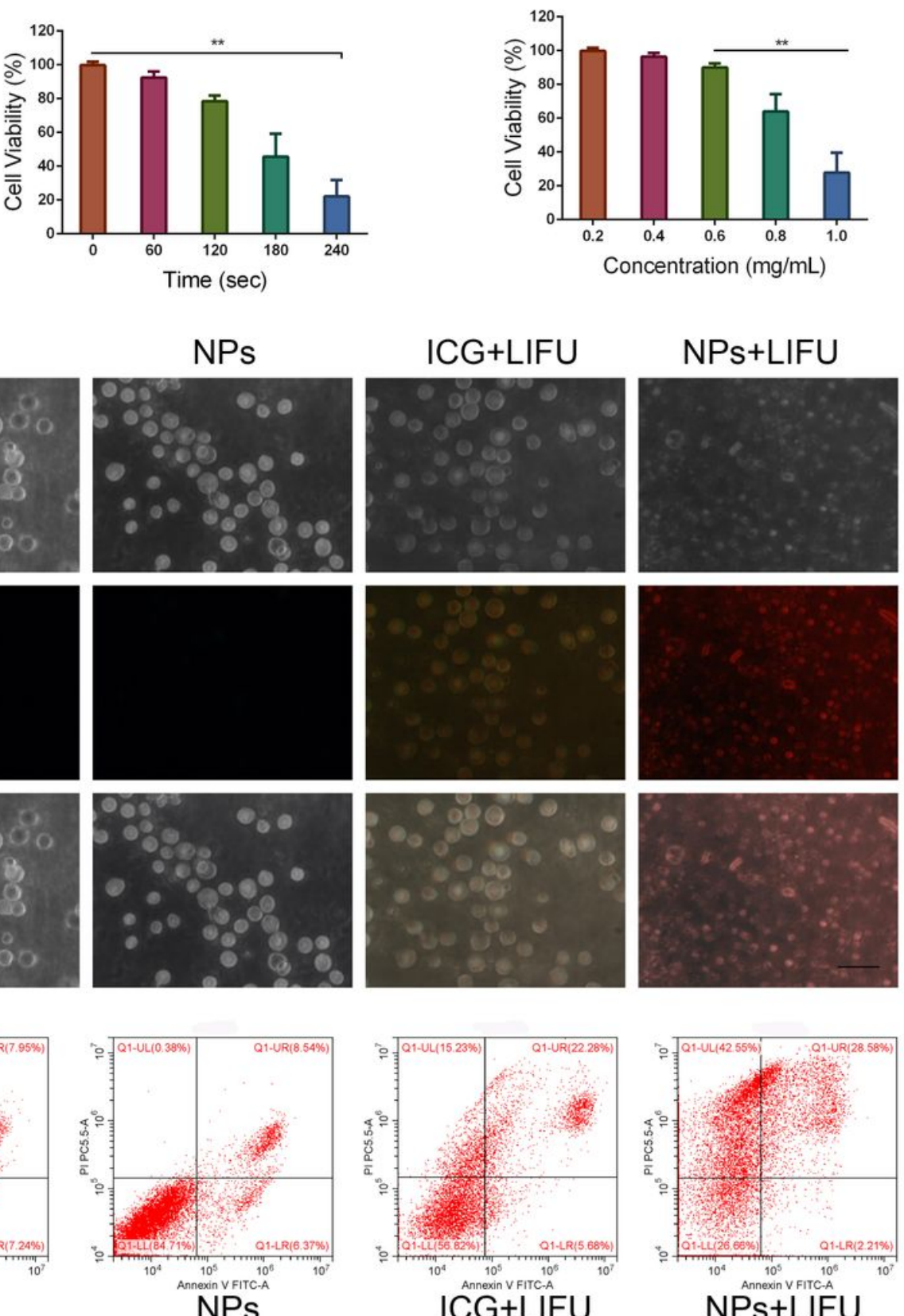

NPs

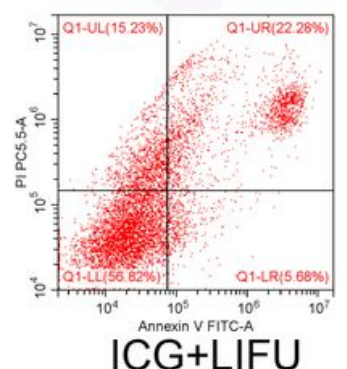

ICG+LIFU

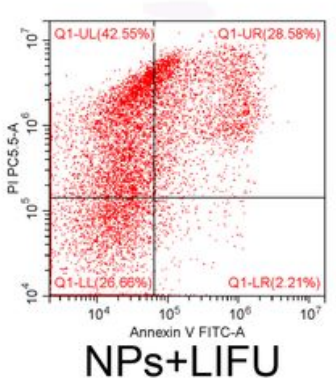

Figure 5

Intracellular ROS generation capacity of Lip-ICG-PFP-cRGD. (A) CLSM images and (B) flow cytometry analysis of ROS generation in ID8 cells treatment with various treatments, as detected with DCFH-DA. 
A

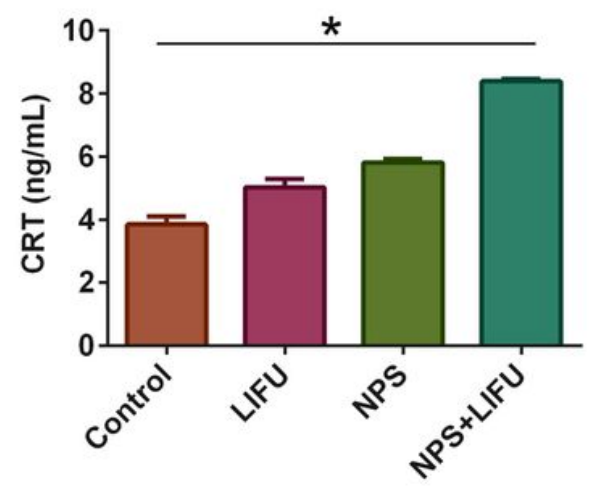

C

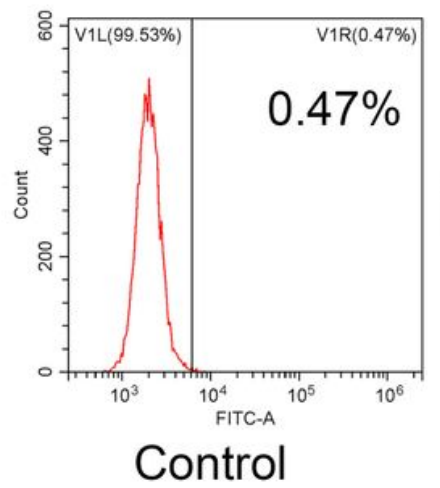

B

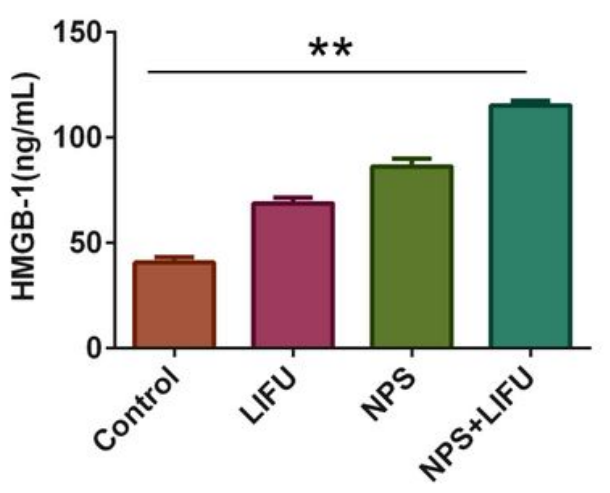

Figure 6

(A) Relative cell viability of ID8 cells after incubation with different concentrations of Lip-ICG-PFP-CRGD. $(B, C)$ Relative cell viability of ID8 cells after various treatments. (The error bars represent standard deviation, $n=3$ ). (D) Fluorescence microscope image of annexin V/PI-stained cells. (E) Flow analysis of apoptosis of ID8 cells after various treatments. 
A

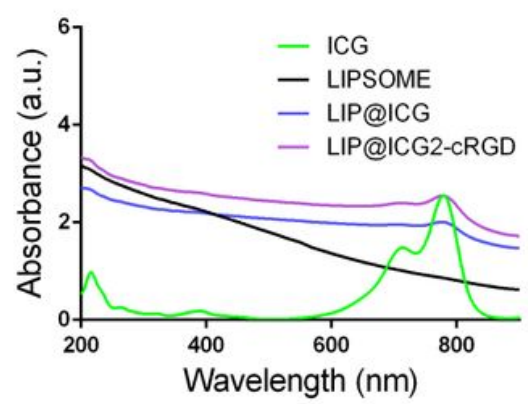

D

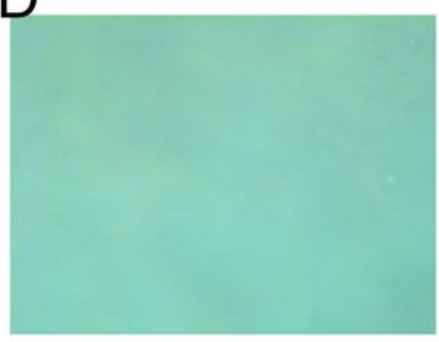

Before
B

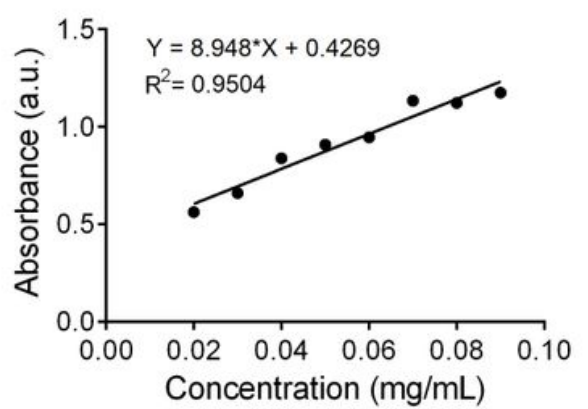

C

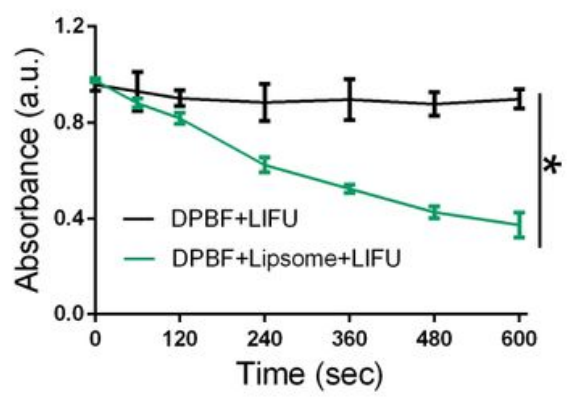

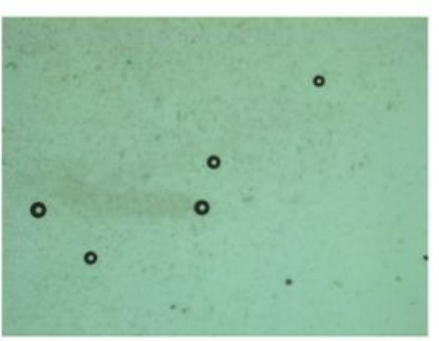

$2 \mathrm{~W}$

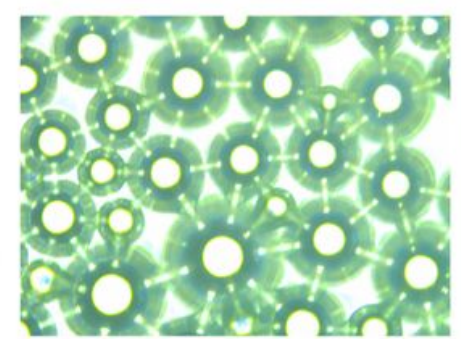

$4 \mathrm{~W}$
$50 \mu \mathrm{m}$

$6 \mathrm{~W}$

\section{Figure 7}

(A, B) Quantitative assay of CRT exposure and release of HMGB1 into the medium using ELISA. (The error bars represent standard deviation, $n=3$ ). (C) The amount of CD86+ DCs through flow cytometry after various treatment in vitro.

\section{Supplementary Files}

This is a list of supplementary files associated with this preprint. Click to download.

- Graphicalabstractimage.jpg 